\title{
Los mapas de posicionamiento: una alternativa para determinar competidores
}

\author{
Positioning maps: an alternative to define competitors
}

Zoila Ulacia Oviedo. ${ }^{~,}$, Dailyn Sánchez Rego. ${ }^{2}$, Suleena Céspedes Pavot. ${ }^{3}$, Juan Ernesto Gutiérrez Leyva. ${ }^{4}$ \& Luis Efraín Velastegui López. ${ }^{5}$

Recibido: 13-03-2021 / Revisado: 20-03-2021 /Aceptado: 10-04-2021/ Publicado: 05-05-2021

\begin{abstract}
DOI: https://doi.org/10.33262/concienciadigital.v4i2.1.1707

The increasing levels of competition in the tourism sector today and some more experienced and demanded tourists, force hotels managers to apply judgments of greater professionalism in the management of their establishments, and to pay particular attentionto the satisfaction of the customer needs and to the detection and identification of market opportunities that may arise. This study proposes the identification of the Hotel Santa Isabel's competitors, by studying its position in the market. To accomplishthe definition of the competitors, a change of approach of the positioning studies was made, starting from the design of a perceptual map or market positioning map. The change of approach was given under the conditions of considering a competitor of an establishment, to that company that delivers the same attributes to the same market segment in a similar way. As a result, was determined the main competitor of the Hotel Santa Isabel, based on its location on a three-dimensional positioning map and the distance in relation to the rest of the hotels studied, demonstrating the usefulness of this tool for identifying competitors.
\end{abstract}

Key Words: Positioning; Competitors; Perceptual Map; Attributes; Heritage Hotels

\footnotetext{
1 Universidad de la Habana, Facultad de Turismo, La Habana, Cuba, email: zoila@ftur.uh.cu: ORCID:0000-0002-8818-083x

2 Universidad de la Habana, Facultad de Turismo, La Habana, Cuba,

3 Universidad de la Habana, Facultad de Turismo, La Habana, Cuba, email:sul3cespedes@gmail.com, ORCID: 0000-0003-2295-0405

4 Universidad de La Habana, Facultad de Turismo, La Habana, Cuba, email: jernesto@ftur.uh.cu: ORCID: 0000-0002-0506-5685

${ }^{5}$ Ciencia Digital Editorial, Ecuador, luisefrainvelastegui@cienciadigital.org
} 


\section{Resumen}

Los crecientes niveles de competencia que existen hoy en el sector turístico y unos turistas cada vez más experimentados y exigentes, obligan a los gestores hoteleros, a aplicar criterios de mayor profesionalidad en la gestión de sus establecimientos y a poner un mayor énfasis en la satisfacción de las necesidades de la clientela y en la detección e identificación de las oportunidades de mercado que puedan presentarse. Este estudio tiene dos objetivos principales: 1) identificar los competidores del Hotel Santa Isabel, mediante el estudio de su posicionamiento en el mercado, a los fines de contribuir al diseño de sus estrategias de consolidación dentro del mismo y 2) demostrar la validez de los mapas de posicionamiento para la identificación de competidores. La idea rectora fue concebida sobre la base del criterio de que un estudio de posicionamiento, permite identificar los competidores de una Organización, contribuyendo a sus estrategias de consolidación en el mercado. Para el trabajo, se realizó un cambio de enfoque de los estudios de posicionamiento, sustentándose dicho enfoque en la premisa de considerar competidor de una entidad hotelera, a aquella que entrega, de manera similar y al mismo segmento de mercado, los mismos beneficios. Como resultado del trabajo, se determinó el principal competidor del hotel Santa Isabel, partiendo de su ubicación (en un mapa de posicionamiento tridimensional) y de la distancia respecto al resto de los hoteles estudiados, demostrando la validez y utilidad de esta herramienta en la identificación de competidores.

Palabras claves: Posicionamiento, Atributos; Competidores; Mapa Perceptivo; Hoteles Patrimoniales

\section{Introducción}

El sector turístico en la actualidad está caracterizado por una agresiva competencia entre las empresas. Un acelerado crecimiento de la oferta turística ha provocado que esta supere con mucho, a la demanda. Por otro lado, los consumidores tienen exigencias cada vez mayores y buscan productos que integren todos los beneficios que ellos demandan. Por tanto, las empresas turísticas tienen como reto la puesta en práctica de estrategias de desarrollo que les posibiliten un mejor posicionamiento y una mayor expansión en el mercado.

Las empresas turísticas cubanas forman parte también de este convulso entorno competitivo. Los nichos de mercado de un hotel cubano coinciden con los de muchos otros hoteles en cualquier parte del mundo, en particular, Latinoamérica y el Caribe. Por ello, estos deben empeñarse en ocupar un lugar diferenciado en la mente del cliente, por lo que, deben también, enfocarse en analizar el lugar que ocupan en el proceso de conocimiento, consideración y uso de la oferta.

El posicionamiento, visto como elemento asociado a la mercadotecnia en el sector empresarial, ha sido estudiado y referenciado por muchos autores. Para DiMingo (1988), es el proceso de distinguir una empresa o un producto de sus competidores, con base en dimensiones reales (productos o valores corporativos que sean significativos 
para los consumidores), de modo que, la empresa o producto se torne el preferido en el mercado.

Para Toledo \& Hemzo (1991), el posicionamiento consiste en el desarrollo de una identidad corporativa o de producto distinta,... con el objetivo de predisponer favorablemente al consumidor para que tome la decisión de compra deseada por la empresa. Para estos autores, el punto clave del término en cuestión radica, fundamentalmente, en la imagen que la empresa brinde del producto- servicio que oferta al consumidor. Por otro lado, no consideran importante el lugar que pueda ocupar el producto de acuerdo a esta imagen.

Kotler y Armstrong (2017), aportan una definición más clara y completa, al asumir que la posición de un producto es la forma como los consumidores lo definen, de acuerdo con atributos importantes. Es el lugar que el producto ocupa en la mente del consumidor, en relación con los otros productos de la competencia. Este autor define como elemento de importancia, el lugar que tiene un producto-servicio para el cliente, dada la subjetividad que pueda existir en la mente del individuo. Además, hace referencia a los atributos como elementos determinantes para la posición del producto.

Según (Fischer, 2011) el atributo es un adjetivo polarizante de un producto- servicio. Sin embargo, otros autores consideran que atributo son aquellos aspectos de la oferta que son valorados por el mercado y que contribuye a que la empresa sea definida de un modo determinado para el cliente. Luego pudiera definirse el atributo como aquellas características que tiene la instalación vinculadas a los beneficios que busca el cliente. Se hace necesario resaltar que los atributos varían de acuerdo al nivel de importancia que le puedan conceder los clientes en su subjetividad. Sin embargo, existen una serie de atributos que pueden resultar comunes para todas las personas partiendo de las necesidades básicas del ser humano, como por ejemplo son la seguridad, la actitud, el costo, entre otros. (Fischer, 2011).

Además de los atributos considerados como universales, existen una serie de atributos que se ajustan a las prioridades y necesidades específicas de cada segmento de mercado. Estos tributan a factores psicosociales como expectativas, percepciones y satisfacción. En consecuencia, también forman parte indisoluble del proceso de valoración del producto- servicio por parte del cliente. Al mismo tiempo, son un factor clave en la conceptualización del producto-servicio y la evaluación de la competitividad de la entidad en cuanto al cumplimiento de los mismos (Ulacia, 2017). En definitiva, los atributos identifican a la entidad e incluso lo diferencian de la competencia.

En el contexto hotelero, dos atributos fundamentan muy claramente, el vínculo de los atributos con el Concepto del producto, a juicio de los autores, Estos son la ubicación y la seguridad. E. M. Statler, el hotelero más famoso de Estados Unidos a principios del siglo XX, declaró en una ocasión que tres de los aspectos más importantes para el éxito de un hotel eran: "ubicación, ubicación y ubicación". Para Statler solo existe un atributo fundamental del producto hotelero. Si bien esto es cierto, somos del criterio de que es 
importante también considerar la seguridad como otro atributo fundamental, atendiendo a los momentos actuales que vive el mundo.

Los atributos percibidos por el cliente, parten de los beneficios que estos buscan en el consumo del producto- servicio en cuestión. Son precisamente los atributos los que provocan que la oferta de cada empresa ocupe un lugar en la mente del cliente, de modo que sean los que posicionen un producto- servicio en el mercado (Carvajal, 2000). Frente a un grupo de hoteles, en el que todos entregan al mismo segmento de cliente el mismo atributo, suele existir un líder que se diferencia del resto. Al mismo tiempo, en orden de prioridad para el cliente, además del líder, unos hoteles lo hacen mejor que otros. Así, cada entidad ocupa un lugar en la mente del consumidor, que depende del grado de efectividad con que esta sea capaz de entregar el atributo más apreciado por el cliente (Llevat, 2011). Ese grado de efectividad puede hacerse reconocer como competitividad.

Porter (1998), interpreta la competitividad como la capacidad de una empresa de obtener ventajas competitivas sostenidas frente a sus rivales en un ambiente de elevada competencia, logrando mayor rentabilidad y creciendo más que estos al conquistar más consumidores e incluso arrebatarle su propia clientela a través de la entrega de mayor valor.

Existen diferentes enfoques metodológicos para realizar estudios de posicionamiento. Uno de estos enfoques es el propuesto por Serra (2003), quien se plantea, como punto de partida, el conocimiento por parte de la empresa, de cómo perciben los consumidores su producto, de acuerdo a una serie de preferencias que estos tengan y en relación a otros productos o marcas competitivas. En función de ello, el autor sugiere el empleo de un mapa perceptual donde se reflejen dichas percepciones. La metodología en cuestión, se muestra en la figura 1.

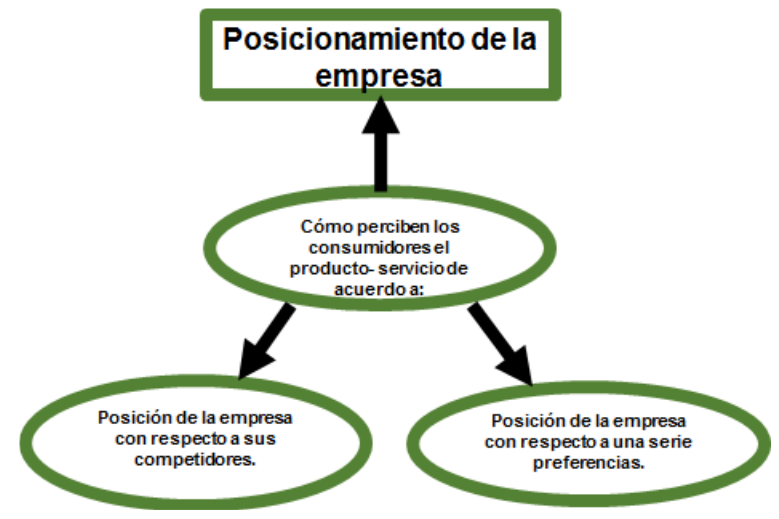

Figura 1 Metodología para un estudio de posicionamiento según Serra (2003)

Kotler (1996), por su parte, basa su metodología sobre la decisión de alternativas de posicionamiento del producto- servicio objeto de estudio. En este sentido, el autor plantea la existencia de seis alternativas: 
1. Posicionamiento por atributo: cuando una empresa establece su posicionamiento con base en el desempeño del producto, según atributos específicos, tangibles o abstractos.

2. Posicionamiento por beneficios: además del resultado del desempeño del producto respecto a ciertos atributos, también tiene en cuenta los beneficios que este aporta al cliente.

3. Posicionamiento por uso/aplicación: presenta el producto como el más apropiado para ser aplicado o usado en una situación específica.

4. Posicionamiento por usuario: asocia el producto a una determinada categoría de usuario, con base a características como estilos de vida, rasgos de la personalidad, historia de vida, etc.

5. Posicionamiento por competidor: compara, de forma implícita o explícita, su producto o marca con el de un competidor, esperando situarse como una mejor alternativa que la del competidor

6. Posicionamiento por categoría de producto: El producto es posicionado como líder en determinada categoría de producto (es más usado cuando se trata de nuevos productos) a partir de enfatizar las diferencia entre las características del producto en cuestión y los restantes del mercado.

Vale la pena señalar que cuando se analiza el posicionamiento de una empresa o un producto, se tienen en cuenta aquellos beneficios que el consumidor espera recibir del producto-servicio. Sin embargo, cuando se realiza un estudio de posicionamiento teniendo en cuenta los atributos, lo que se valora es cómo la empresa entrega esos beneficios al cliente. Por lo anterior, se puede afirmar que el posicionamiento por atributos trasciende al posicionamiento por beneficios, resultando más completo el análisis.

Un tercer exponente de tema en cuestión lo son los autores Trout \& Rivkin (1996), los cuales se suman al enfoque de alternativas de Kotler, pero explicitan una metodología para el estudio del posicionamiento resumida en cuatro pasos: (1) Identificación del atributo más significativo del producto; (2) Conocimiento de la posición de los competidores respecto a dicho atributo; (3) Decidir la estrategia a tomar en función de las ventajas competitivas y (4) Comunicar el posicionamiento al mercado a través de la publicidad. En función del tercer paso, los autores de referencia definen tres alternativas posibles para competir a través del posicionamiento:

- Fortalecer la posición actual en la mente del consumidor.

- Apoderarse de la posición desocupada.

- Desposicionar o reposicionar a la competencia

En esencia, los tres enfoques metodológicos citados anteriormente apuntan a que el posicionamiento es visto como la imagen que tienen los clientes de un producto- 
servicio frente a la competencia. Entonces, cuando se decide efectuar un estudio de posicionamiento, el estudio de la competencia resulta un elemento clave. La posición de un producto no tendría sentido en la mente del cliente si no existieran puntos de comparación con otros productos. De lo que se trata, entonces, es de un fenómeno conocido como competencia. Sobre ello se apunta que existe competencia cuando el consumidor estima que varios productos-servicios ofrecen atributos semejantes, satisfacen igual necesidad o compiten por el mismo presupuesto (Atlantic International University, 2002).

Los resultados de este tipo de estudio, se reflejan en los llamados Mapas de posicionamiento. Esta herramienta resulta muy útil para representar de forma gráfica la situación de la empresa o producto respecto a la competencia en función de determinados criterios. Estos ayudan a conocer la posición actual de una empresa en el mercado para adoptar estrategias de marketing (Carmona, 2010).

Los mapas de posicionamiento permiten hacer un análisis competitivo, donde se identifican las posiciones existentes de los productos de la empresa en cuestión y los de la competencia. Estos análisis se ponen en práctica cuando se planifica una posición para un producto, el cual puede ser el actual de una empresa o uno a lanzar nuevo en el mercado. Después de identificadas las posiciones a través del mapa, generalmente, se elige la estrategia o alternativa de posicionamiento más adecuada y se aplica (Ñanco, 2013).

Aunque existen disímiles formatos para un mapa de posicionamiento, el más usado es el compuesto por un eje vertical y otro horizontal, que se cruzan. En los extremos de los ejes se sitúan el valor máximo y mínimo de cada parámetro a utilizar. La precisión de la información depende de cuan exacto sean los datos de los que disponemos. Aunque estos no sean exactos, la forma más simple, es situarlo sobre la matriz de acuerdo a la información que tengamos del mercado. Una mayor rigurosidad en este sentido, sería la aplicación de encuestas a una muestra representativa del mercado con el que opera la empresa. Esto posibilita que sean los clientes los que precisen la información.

El mapa de posicionamiento se elabora frecuentemente cuando la empresa atraviesa la etapa de planificación de su estrategia de marketing (Carballada, 2011).

Relativo al vínculo posicionamiento-competencia y sobre la base de los análisis anteriores, puede afirmarse que es posible un cambio de enfoque en dicha relación. Los estudios de posicionamiento parten de un entorno competitivo, donde se determina la posición del producto- servicio respecto a la competencia, desde la perspectiva de los clientes. No obstante, es posible afirmar, que estos análisis pueden ser enfocados de modo que se determinen no solo el posicionamiento del producto-servicio, sino también, los principales competidores de la empresa.

A partir de la revisión bibliográfica efectuada y a través de la experiencia práctica de los autores, pudo observarse un desarrollo incipiente de estudios de posicionamiento y de competencia en las distintas cadenas hoteleras cubanas. Ello motivó el desarrollo del 
presente trabajo, apropiándose de la teoría y conceptualidad de los estudios convencionales en el tema, para enfocarlos en la búsqueda de competidores. El contexto considerado más adecuado para dicho estudio fue el de los hoteles patrimoniales, tomándose entonces, como referencia, el hotel patrimonial Santa Isabel, en su momento perteneciente a la Compañía hotelera Habaguanex y hoy formando parte del Grupo Hotelero Gaviota.

Como se apuntó, el hotel Santa Isabel, es un hotel de tipo patrimonial, por cuanto entrega al cliente componentes de valor que se traducen en atributos del establecimiento, como: los valores estéticos y artísticos de carácter patrimonial y el valor social como señal de identidad nacional, entre otros. Los hoteles patrimoniales suelen estar asociados a la degustación de la cultura y el patrimonio de la ciudad en que se encuentren. Además, se caracterizan por encerrar en sí, un valor que puede ser de carácter histórico, cultural, político y/o arquitectónico. Vale resaltar que, un elemento cultural necesita ser inicialmente identificado como un bien patrimonial para convertirse en un recurso turístico. De este modo, se argumenta la estrecha relación que existe entre la cultura y el patrimonio de un recurso turístico (Hernández, 2011).

Esta instalación, no realiza estudios de su competencia, por lo que tampoco emplean técnicas ni herramientas encaminadas a ello, reconociendo esta únicamente, de manera empírica, lo que limita la concepción de estrategias de mercado efectivas.

\section{Metodología}

El primer paso a realizar fue la selección del hotel objeto de estudio, así como, los hoteles que pudieran entrar en una comparación con este, partiendo de la decisión previa de la zona turística donde se quería realizar el estudio: la zona histórica patrimonial de La Habana, por cuanto es el territorio habanero con mayores valores y atractivos reconocidos por el turismo extranjero lo que hace que sea el más visitado por este. Ello condujo a estudiar el conjunto de hoteles de la compañía que se encontraban ubicados en dicha zona. El estudio permitió, por una parte, caracterizarlos de modo general y por otra, hacer la elección del hotel a estudiar y sus posibles competidores.

El primer objetivo del estudio permitió concluir que los hoteles ubicados en la zona, suelen estar asociados a la cultura y la historia del lugar, considerados por tanto, hoteles patrimoniales. La mayoría de ellos encierran en sí un rico patrimonio arquitectónico, conservando los rasgos típicos de las mansiones coloniales de la época. Hace más de dos décadas, que la Organización de las Naciones Unidas para la Educación, la Ciencia y la Cultura (UNESCO), le confirió al imponente conjunto arquitectónico del Centro Histórico de La Habana la distinción de Patrimonio Cultural de la Humanidad.

El segundo objetivo del estudio, permitió identificar aquellos hoteles más representativos de la compañía y los de mayor categoría en términos de estrellaje. El estudio también condujo a determinar, mediante el criterio de segmentación de mercados los hoteles que podrían incluirse en un mapa de posicionamiento. El resultado 
fue que solo 4 de los 7 hoteles de mayor categoría, operaban los mismos segmentos, por lo que podrían ser analizados en un mapa de posicionamiento.

La segunda cuestión radicó en hacer, de los cuatro hoteles, la elección del hotel objeto de estudio y sus competidores. De este trabajo, el hotel Santa Isabel resultó ser el idóneo, siendo los 3 restantes los analizados como competidores del mismo.

La idoneidad del hotel Santa Isabel se fundamentó en ser un hotel pequeño (27 habitaciones), una estructura organizativa simple, un reducido número de trabajadores y la no existencia de un departamento comercial (concentrada esta función en la gerencia del hotel). Estas características distintivas facilitarían el trabajo a realizar.

Establecido el contexto del trabajo, se estuvo en condiciones de iniciar las fases de la investigación cuya trayectoria metodológica (Figura 2) estuvo conformada por 3 fases.

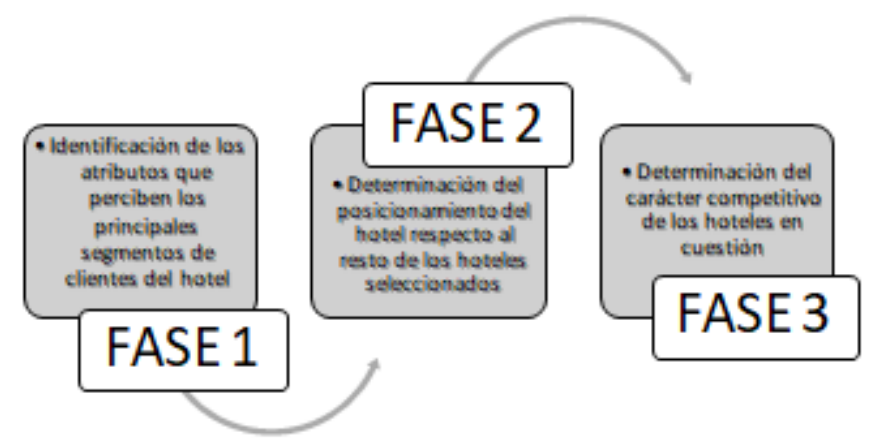

Figura 2. Trayectoria metodológica de la investigación.

\section{Desarrollo de las fases. Resultados}

\section{Atributos percibidos por los principales segmentos de clientes del Hotel Santa Isabel}

Esta fase se inició con la identificación de los principales segmentos que operaba el hotel. Mediante una entrevista a la gerente del hotel, se pudo conocer que los segmentos a los que se orientaba la instalación eran hombres de negocios y parejas, con un rango de edad entre los 51 y 60 años y su principal motivación de viaje era vacacional.

La caracterización de estos segmentos, sobre la base de los atributos más apreciados por ellos, resultó el listado que aparece en la tabla 1. La revisión bibliográfica (documentación referida a segmentación de clientes y determinación de atributos preferidos por cada segmento) y la encuesta (dirigida a turistas que se hospedan en hoteles cercanos a zonas comerciales, entiéndase, la zona del Centro de Negocios de Miramar, la del Vedado cercana al Meliá Cohíba y en la Habana Vieja cercana a la Lonja del Comercio y parejas en zonas turísticas de importancia en la ciudad como el Centro Histórico de la ciudad, Plaza de la Revolución...) fueron los instrumentos empleados para ello. 
Como se aprecia en la Tabla 1, cada segmento difiere del otro en un atributo respectivamente. De esta manera se decidió finalmente, emplear la lista de los nueve atributos como los más apreciados por cada segmento de manera general.

Tabla 1. Atributos más apreciados por los segmentos de mercado en estudio

\begin{tabular}{ccc}
\hline Segmentos & Hombres de negocio & Parejas \\
\hline & Seguridad & Seguridad \\
& Confort & Confort \\
Ubicación & Ubicación \\
Atributos & Tranquilidad & Tranquilidad \\
& Ambientación & Ambientación \\
& Limpieza & Limpieza \\
& Nivel de Servicio del Personal & Nivel de Servicio del Personal \\
& Lujo & Variedad de servicios \\
\hline
\end{tabular}

Con el fin de saber qué atributos son identificados en el hotel y qué evaluación reciben por parte del cliente, se aplicó una encuesta a los clientes del hotel con el listado de atributos obtenido anteriormente. La técnica de muestreo probabilístico aleatorio, se aplicó, dos veces para las dos poblaciones con el objetivo de determinar las muestras representativas: una del total de turistas físicos que se hospedaron en La Habana en ese mismo periodo del año anterior (73) y la otra del total de turistas físicos que se hospedaron en el hotel Santa Isabel en el año anterior del mismo periodo (71). La escala Likert, se aplicó para la evaluación de los atributos en el hotel si estos eran apreciados. El proceso de las encuestas se realizó durante tres meses: enero, febrero y marzo

La información relacionada con la apreciación de los atributos en el hotel Santa Isabel por parte de sus principales segmentos de mercado, se muestra en el gráfico 1, donde se aprecia que todos los atributos son percibidos por los clientes encuestados del hotel.

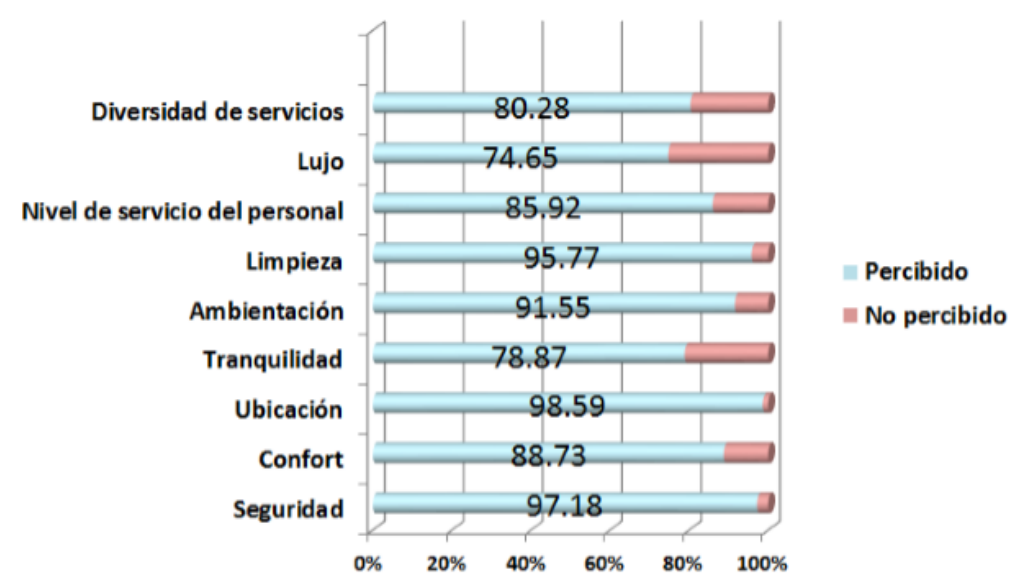

Gráfico 1: Atributos percibidos por los clientes del hotel Santa Isabel.

Determinación del posicionamiento del Hotel Santa Isabel, respecto al resto de los hoteles estudiados.

La determinación del posicionamiento del hotel Santa Isabel, se realizó tomando en cuenta los hoteles que constituían el entorno competitivo más cercano, por ser hoteles 
patrimoniales de alta categoría enfocados a los mismos segmentos de la demanda. Estos fueron Palacio del Marqués de San Felipe, Telégrafo y Terral.

De estos hoteles, resultó necesario conocer, por medio de la encuesta realizada, el perfil demográfico de los principales segmentos de clientes con los que operaba cada entidad, resultando la caracterización de estos en ese sentido, muy similar a los del hotel Santa Isabel (mismos segmentos, motivación de viaje y rangos de edad.

Para la realización del mapa de posicionamiento fue necesario determinar cómo valoraban los clientes la entrega de los atributos ya identificados. El trabajo se realizó a partir de una encuesta a los clientes de cada uno de los hoteles en estudio. La determinación de la muestra en cada uno de ellos se apoyó en un muestreo aleatorio utilizando el mismo procedimiento que los anteriormente usados en el cálculo de la muestra de clientes del hotel Santa Isabel, siendo esto posible ya que el objetivo del uso de la herramienta fue el mismo. La muestra para el hotel Telégrafo fue 71clientes; del hotel San Felipe 70 y del hotel Terral 68. Las evaluaciones obtenidas como resultado de este trabajo se muestran en los siguientes gráficos:
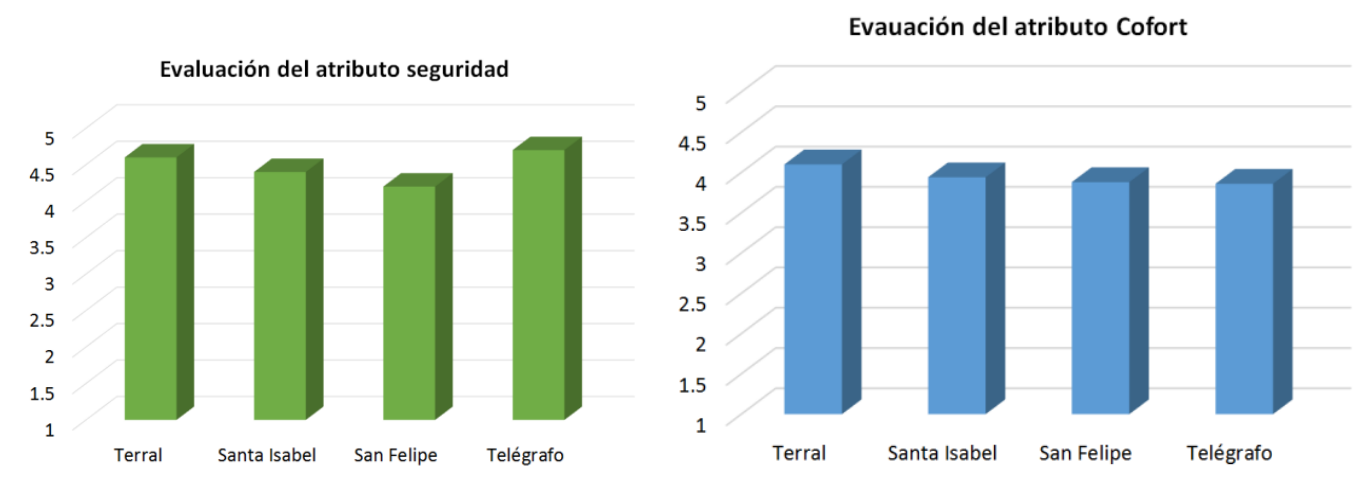

Evaluación del atributo Ubicación

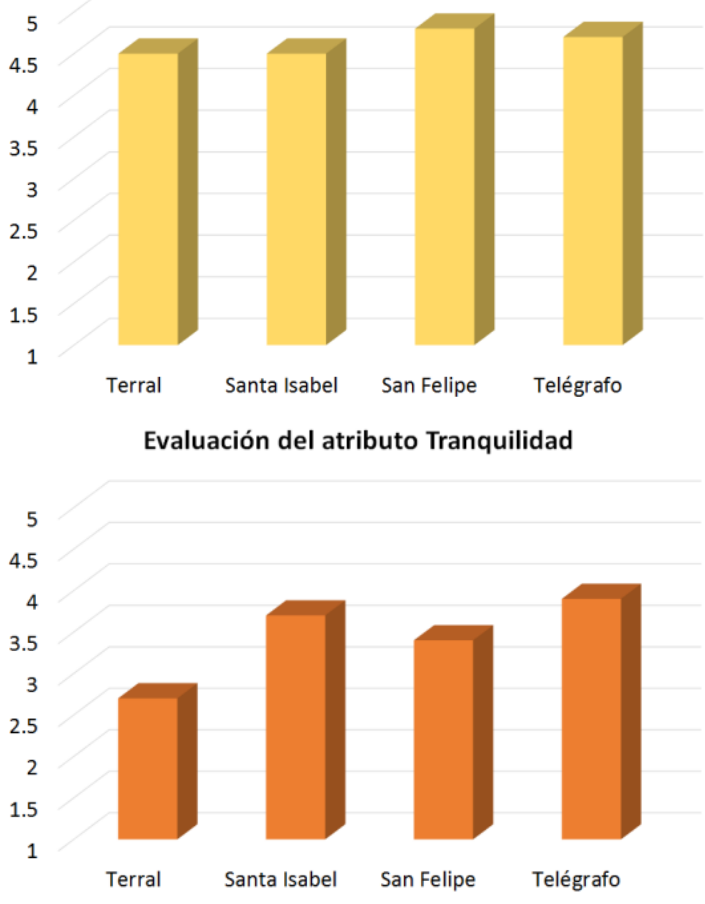




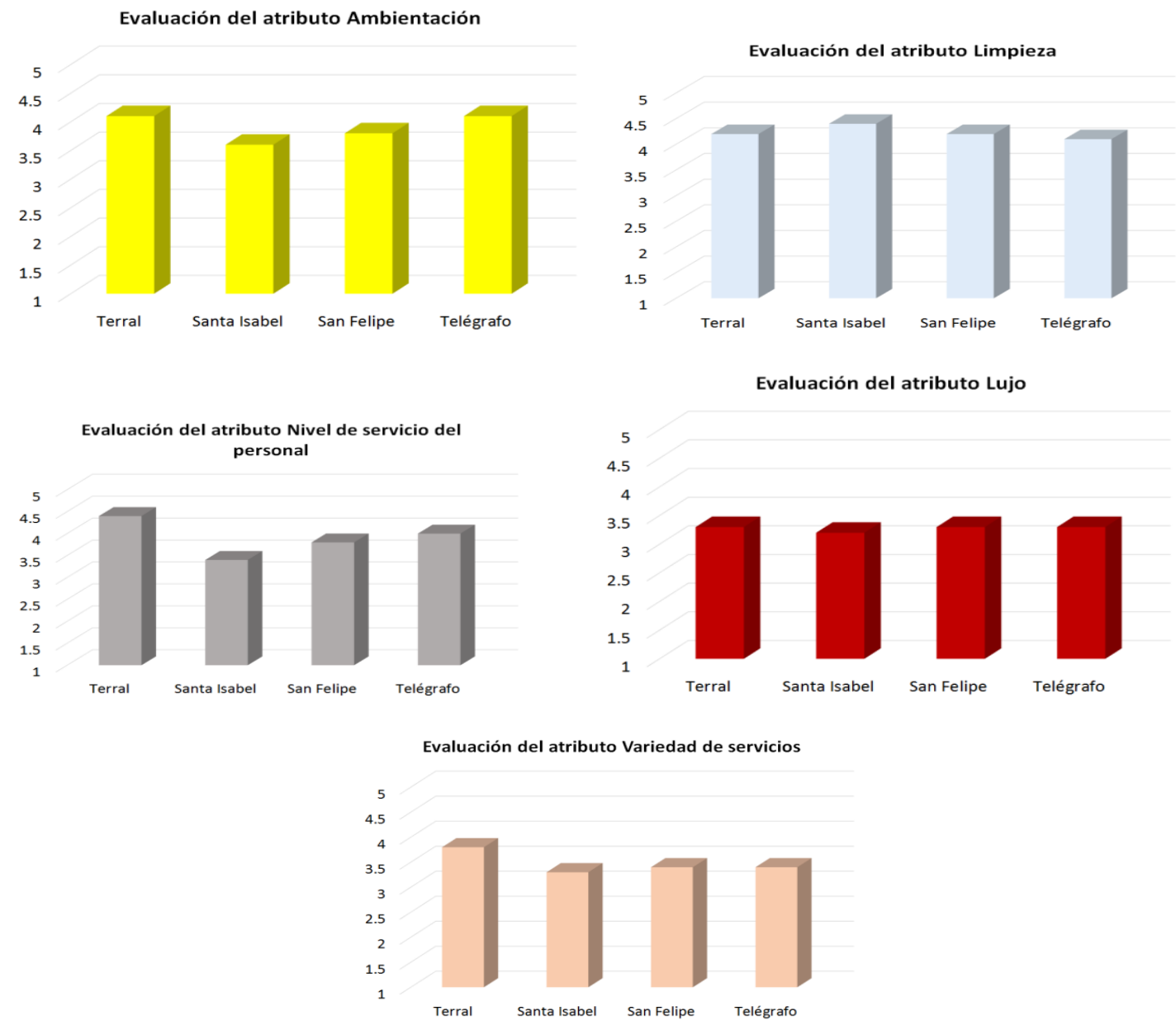

El atributo Seguridad, a pesar de haber tenido evaluaciones similares en los cuatro hoteles, es el hotel San Felipe $(4,24)$ donde la percepción de los clientes es la más baja. El hotel Telégrafo $(4,66)$ resultó el líder en el atributo seguridad, para el segmento de mercado en cuestión.

El atributo Confort es entregado por los cuatro hoteles de manera similar, sin embargo, pudiera reconocerse un ligero liderazgo en este atributo del hotel Terral.

El atributo Ubicación fue percibido como bueno por los clientes en los cuatro hoteles, sin embargo, la mejor evaluación en este sentido la obtiene el hotel San Felipe, siendo la ubicación menos preferida la del hotel Terral, siguiéndole el hotel Santa Isabel.

A diferencia de los analizados anteriormente, el atributo Tranquilidad muestra diferencias significativas en las percepciones del cliente en cada entidad. Se observa que el hotel Santa Isabel es percibido en este atributo, de forma muy similar al líder del mercado (hotel Telégrafo). Teniendo en cuenta las evaluaciones en general, se puede afirmar que el atributo Tranquilidad no es percibido favorablemente por los clientes de los cuatro hoteles. Ello puede estar fundamentado por la ubicación de estos y el hecho de tratarse de hoteles de ciudad, donde la vida es más agitada y activa. 
El atributo Ambientación, fue percibido por los clientes de los cuatro hoteles casi de igual forma. No obstante, las pequeñas diferencias permiten afirmar que el hotel Terral $(4,13)$ resultó ser el líder y el Santa Isabel $(3,65)$ el que entrega el atributo con mayor deficiencia

La Limpieza se percibe de forma satisfactoria en los cuatro hoteles, lo que se sustenta en las pequeñas diferencias en la evaluación recibida por cada uno de ellos.

El Nivel del servicio del personal presenta diferencias en las cuatro entidades. El hotel con mejor nivel de servicio del personal percibido es el Terral $(4,46)$, mientras que el hotel Santa Isabel $(3,41)$ es el de peor evaluación.

El Lujo es un atributo evaluado de mal en todos los hoteles objeto de estudio de manera general, pues las puntuaciones dadas por los clientes oscilan en el rango de 3 a 3,50 sin presentar casi diferencias.

La Variedad de los servicios es percibida por los clientes bastante mal. Las percepciones no presentan casi diferencias

En resumen, los análisis realizados sugieren que existen algunos atributos que no son bien percibidos por los clientes en los cuatro hoteles. Vale resaltar que el hotel Santa Isabel no tuvo un papel destacado en las evaluaciones de los clientes. Este hotel fue líder en solo un atributo: Limpieza. Además, obtuvo la menor puntuación en tres atributos: Ambientación, Nivel de servicio del personal y Variedad de servicios.

Estos resultados permitieron elaborar el mapa de posicionamiento o mapa perceptual, como también suele conocérsele. Este es una vía gráfica mediante la cual se pueden presentar las percepciones del consumidor acerca de un producto-servicio de una empresa respecto a su competencia. Sin embargo, la técnica fue empleada en esta fase, con el fin de determinar los principales competidores del Hotel Santa Isabel. Este cambio de enfoque se centra en dos condicionantes esenciales: (1) existe competencia si los productos- servicios en cuestión, entregan los mismos beneficios a un mismo segmento de clientes y (2) se entregan los atributos de estos productos-servicios de manera similar. Cuando se dan estas dos condicionantes, entonces existe un entorno competitivo entre las empresas.

A pesar de tratarse de dos segmentos de clientes, la información fue unificada y tratada globalmente, atendiendo a que el segmento de mercado de hombres de negocios constituye solo una parte muy pequeña del total de encuestados, lo cual demuestra que aunque en las instalaciones se habla de una segmentación definida, en la práctica no ocurre así.

Pese a que un mapa de posicionamiento por lo general se limita a dos atributos, se consideró que un mapa tridimensional, donde se reflejen tres atributos, sería más conveniente. El mapa tridimensional refleja la posición de cada hotel en la mente del consumidor, es decir, la posición que ocupan estos para los nichos de mercados encuestados. 
El diseño de este tipo de mapa, consta de tres ejes o dimensiones, los cuales representan un atributo por separado. Cada eje consta de una escala de 1 a 5 (escala Likert), en correspondencia a la escala empleada en el cuestionario para evaluar los atributos. Los puntos que se reflejan en el mapa muestran la posición de cada hotel y las coordenadas de cada punto respecto a los ejes son resultado de las valoraciones hechas de cada atributo, por parte de los clientes. Existieron tantos puntos en el mapa diseñado como hoteles en cuestión, en este caso cuatro (4).

Para su elaboración se consideró el empleo de un programa matemático (MATLAB) que, a través de la inserción de los datos proporcionara mayor exactitud en el diseño y la información que se quería reflejar.

Vale referir que, en toda la literatura revisada aunque se hace referencia a los mapas tridimensionales, no se encontró ningún ejemplo práctico de los mismos. De este modo se pensó que sería novedoso poner en práctica un mapa de posicionamiento tridimensional, además de que este modelo de mapa, ofrece mayor información unificada del producto permitiendo interpretar simultáneamente, la posición de las empresas en cuanto a tres dimensiones.

Para determinar el posicionamiento de cada entidad, además de lo observado en el mapa, se calcularon las distancias entre los puntos. Lo anterior permitió tener una mayor precisión de la posición ocupada por cada hotel en la mente del cliente en cuanto a los atributos en cuestión. Las distancias se calcularon través de la fórmula matemática, que se deriva del principio de la llamada distancia euclidiana que parte del Teorema de Pitágoras:

$$
d=\sqrt{\left(H 1_{a 1}-H 2_{a 1}\right)^{2}+\left(H 1_{a 2}-H 2_{a 2}\right)^{2}+\left(H 1_{a 3}-H 2_{a 3}\right)^{2}}
$$

Los mapas y la determinación de las distancias entre los puntos para determinar el posicionamiento de cada entidad con respecto al hotel Santa Isabel, se desarrollaron con el asistente matemático ya referido con anterioridad.

El posicionamiento que tiene el hotel Santa Isabel respecto a los restantes hoteles se llevó a cabo mediante el diseño de tres mapas tridimensionales. Cada uno de estos mapas permite apreciar una posición para cada hotel teniendo en cuenta tres atributos diferentes en cada mapa (Figuras 3, 4 y 5).

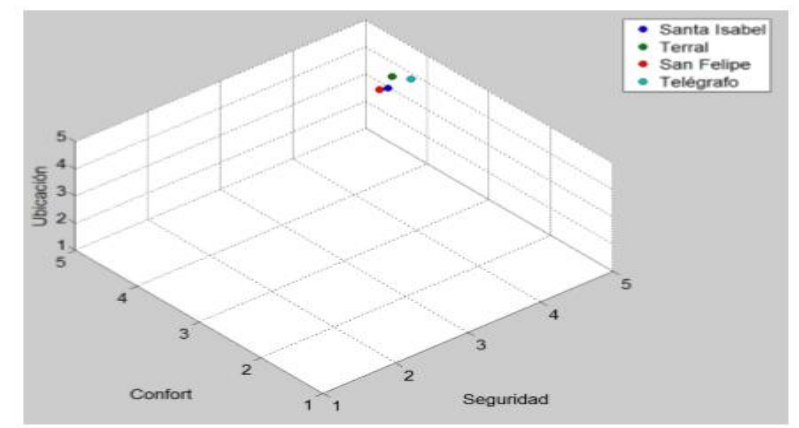

Figura 3. Mapa de Posicionamiento respecto a los atributos Seguridad, Confort y Ubicación. 
Como se aprecia en el mapa anterior, las distancias entre los cuatro hoteles son pequeñas, de modo que estos tienen una posición similar, aunque es posible afirmar que existen algunas diferencias entre ellos que los posicionan de manera diferente en el mercado consumidor. No existe un hotel mejor posicionado en estos tres atributos simultáneamente, dado que las distancias entre los puntos son muy pequeñas y por tanto, las diferencias entre las puntuaciones son mínimas. Sin embargo, el hotel Telégrafo, Terral y San Felipe son los líderes de los atributos seguridad, confort y ubicación respectivamente. El Hotel Santa Isabel además de no resultar líder en ninguno de estos atributos, tampoco estuvo cerca del líder en ninguno de las tres puntuaciones por separado.

Para comprobar la afirmación anterior se calcularon las distancias entre el hotel Santa Isabel y cada uno de los otros hoteles. Los resultados obtenidos fueron:

$\checkmark$ Santa Isabel/Telégrafo $=0.307409$

$\checkmark$ Santa Isabel/Terral $=0.298998$

$\checkmark$ Santa Isabel/San Felipe $=0.292233$

El segundo nivel de análisis en el estudio de posicionamiento, abordó otros tres atributos: Limpieza, Ambientación y Tranquilidad (Gráfico 4). En este caso, las distancias son mayores y las posiciones están mejor definidas según muestra el gráfico. El hotel más alejado del resto, en esta posición del mapa, es el Hotel Terral, debido a la puntuación tan baja (en la escala de Likert) que obtuvo en el atributo Tranquilidad. Los líderes en este caso fueron Telégrafo, San Felipe y Santa Isabel para los atributos tranquilidad, ambientación y limpieza, respectivamente.

Lo anterior se confirma a través del cálculo de las distancias entre el hotel Santa Isabel y los restantes hoteles:

$\checkmark$ Santa Isabel/Telégrafo $=0.534228$

$\checkmark$ Santa Isabel/Terral $=1.170299$

$\checkmark$ Santa Isabel/San Felipe $=0.441135$

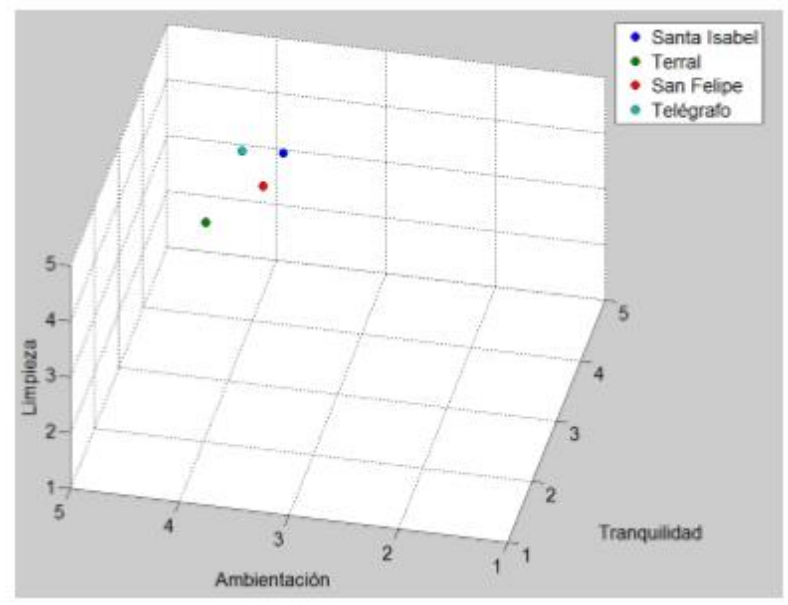

Figura 4. Mapa de Posicionamiento respecto a los atributos Limpieza, Ambientación y Tranquilidad 
Un tercer nivel de análisis responde al mapa de posicionamiento que estudia los atributos Nivel del personal de servicio, Lujo y Variedad de los servicios (Gráfico 5).

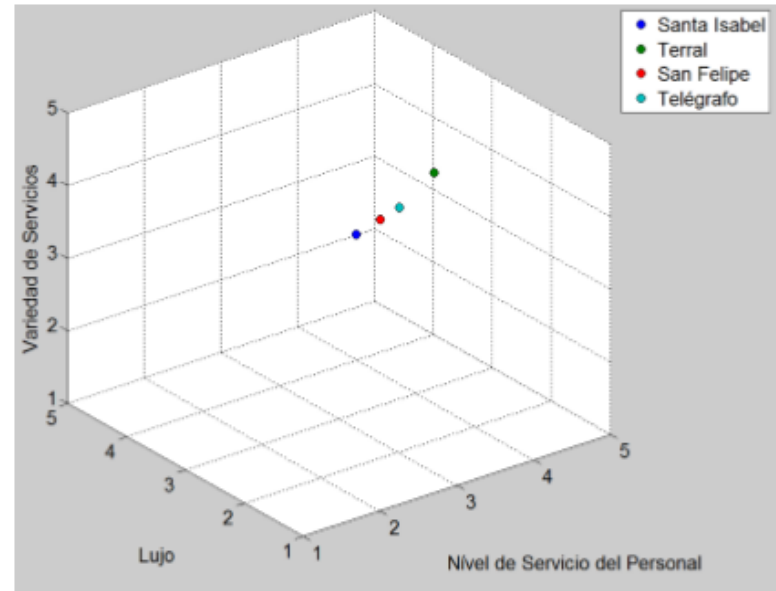

Gráfico 5. Mapa de Posicionamiento respecto a los Atributos Lujo, Variedad de los Servicios y Nivel de servicio.

Como se puede observar, el hotel peor posicionado en este caso es el hotel Santa Isabel. La mejor posición la ocupa el Hotel Terral, el cual se convierte en el líder de estos tres atributos. Es este el único caso en el que un solo hotel es líder para los tres atributos y por tanto, es el mejor posicionado para el segmento de mercado que se estudia. El cálculo de las distancias demuestra lo anteriormente afirmado:

$\checkmark$ Santa Isabel/Telégrafo $=0.607701$

$\checkmark$ Santa Isabel/Terral $=1.147432$

$\checkmark$ Santa Isabel/San Felipe $=0.358608$

Finalmente, se realizó un cálculo total de las distancias, para determinar entonces una posición global para el Hotel Santa Isabel basada no solo en los mapas sino en el cálculo. Siendo los resultados:

$\begin{array}{ll}\checkmark & \text { Santa Isabel/Telégrafo }=0.865563 \\ \checkmark & \text { Santa Isabel/Terral }=1.666013 \\ \checkmark & \text { Santa Isabel/San Felipe }=0.639218\end{array}$

Los resultados de las distancias de cada mapa y, por ende, los globales apuntan a que es el Hotel San Felipe el más cercano al Hotel Santa Isabel. Por ello, las posiciones que ocupan ambas entidades en la mente del consumidor son muy similares. No obstante, es apreciable en cada mapa que el Hotel Telégrafo se encuentra también cercano al Santa Isabel. Las distancias tienen diferencias mínimas y aunque no es el más inmediato en cuanto a cercanía, también tiene una posición muy similar.

Por otro lado, es apreciable que el hotel Terral se mantuvo más alejado. Sin embargo, la lejanía significó un posicionamiento ventajoso, pues es este hotel el que tiene una mejor 
posición de manera general. Esto, permite afirmar que el hotel Terral es el líder en el estudio realizado.

\section{Determinación de los competidores del Hotel Santa Isabel.}

La cuarta y última fase tuvo como fin determinar cuáles son los principales competidores del Hotel Santa Isabel, partiendo del enfoque asumido para el empleo del Mapa de Posicionamiento. Para ello, fue necesario tener en cuenta que el carácter competitivo está dado por la cercanía entre los puntos reflejados en el mapa (cada punto constituye un hotel). Los puntos más cercanos al hotel Santa Isabel representan aquellos hoteles que entregan al mismo segmento de mercado, los mismos atributos de manera muy similar. Se consideró que cuando se cumplen ambas condicionantes se estaba en presencia de un competidor de la entidad. En conclusión, mientras más cerca se encuentre el hotel Santa Isabel a otros hoteles, mayor es el carácter competitivo entre ellos.

El cálculo de las distancias entre los puntos, constituyó el criterio determinante en la cercanía y por tanto en el carácter competitivo entre los hoteles. De este modo, resultó que para el segmento de mercado de turistas con edades entre 51 y 60 años, que viajan en parejas fundamentalmente y que tienen como principal motivación de viaje vacacionar, el hotel Santa Isabel tiene dos competidores: el hotel San Felipe y el hotel Telégrafo. El primero, resultó ser el principal competidor en tanto se encuentra más cerca en el posicionamiento analizado, siguiéndole en cercanía y en consecuencia, ocupando el segundo lugar, el hotel Telégrafo.

Vale resaltar que las distancias entre el hotel Santa Isabel y los dos competidores son muy pequeñas, lo que evidencia que las evaluaciones de los atributos, hechas por el segmento de clientes en cuestión, de manera general, se mantuvieron muy cercanas para las tres entidades.

\section{Conclusiones:}

- Los estudios de posicionamiento en el mercado desde la perspectiva de los atributos del producto-servicios, presentan elementos de análisis más abarcadores, posibilitando la determinación de los competidores. El diseño de un mapa de posicionamiento tridimensional, permitió tener una visión más amplia y precisa en este sentido.

- El estudio de posicionamiento en el mercado del hotel Santa Isabel, permitió identificar sus competidores, entendiéndose como competidor, aquella entidad que opera el mismo segmento de mercado y la entrega similar de los mismos atributos.

- Los competidores del hotel Santa Isabel son los hoteles San Felipe y Telégrafo, siendo el principal el hotel San Felipe. 
- El principal segmento de mercado que opera el hotel Santa Isabel (parejas de turistas vacacionistas con edades que oscilan entre 51 y 60 años, percibe nueve atributos del producto: Seguridad, Confort, Ubicación, Tranquilidad, Ambientación, Limpieza, Nivel de servicio del personal, Lujo y Variedad de servicios.

- El posicionamiento del hotel Santa Isabel evidencia que este ocupa una posición alejada del líder del mercado: el hotel Terral.

\section{Bibliografía:}

Atlantic International University. (2002). Competitive Strategy.

Carballada, C. P.,( 2011). 7 Pasos para Posicionar una Marca o Producto. [En línea] Available at: http://marketisimo.blogspot.com/2010/02/7-pasos-para-posicionaruna-marca-o.html

Carmona, O. (2010). Los mapas perceptuales (perceptual mapping) y su aplicación al mercadeo. Obtenido de La investigación de mercados hoy: http://ocarmona.blogspot.com/2010/03/los-mapas-perceptuales-y-su-aplicación

Carvajal G. G., 2000. Posicionamiento: El caso Latinoamericano. Bogotá: Mc Graw Hill Interamericana, S. A.

DiMingo, E. (1988). The fine art oh positioning. The journal of Business Strategy, 3438.

Fischer, R. (2011). El desafío del Servicio. Versión 2.0 Hospitality \& Service University. Santiago de Chile

Hernández, G., 2011. Alemania, potencialidades para el turismo cultural en la Habana, La Habana: Facultad de Turismo. Universidad de La Habana.

Kotler, P., 1996. Marketing Management: Analysis, Planning and Control. U.S.A.: Prentice Hall.

Llevat, M. (2011). Premisas para el rediseño de la gestión del hotel Victoria bajo los preceptos de la gerencia del servicio. La Habana: Facultad de Turismo, Universidad de La Habana.

Ñanco, S., 2013. Construcción Mapa de Posicionamiento. [En línea] Available at: http://es.slideshare.net/snanco/construccion-mapa-de-posicionamiento

Porter, M. (1988). Ventajas Competitivas. Boston: Free Press. 
Toledo, G. \& Hemzo, M., (1991). El proceso de posicionamiento y el marketing estratégico. Belo Horizonte, s.n.

Trout, H.; Rivkin, P., 1996. El nuevo Posicionamiento. Ed. Limusa. Mexico:

Ulacia, Z. (2015). Sistemas de Servicio y Hospitalidad. Edit. Universitaria Félix Varela. La Habana

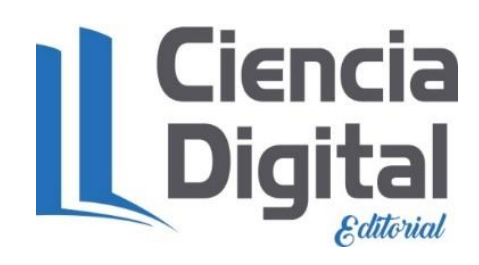




\section{PARA CITAR EL ARTÍCULO INDEXADO.}

Ulacia Oviedo, Z., Sánchez Rego, D., Céspedes Pavot, S., Gutiérrez Leyva, J. E., \& Velastegui López, L. E. (2021). Los mapas de posicionamiento: una alternativa para determinar competidores. ConcienciaDigital, 4(2.1), 62-80. https://doi.org/10.33262/concienciadigital.v4i2.1.1707

\section{LCiencia}

El artículo que se publica es de exclusiva responsabilidad de los autores y no necesariamente reflejan el pensamiento de la Revista Ciencia Digital.

El artículo queda en propiedad de la revista y, por tanto, su publicación parcial y/o total en otro medio tiene que ser autorizado por el director de la Revista Ciencia Digital.

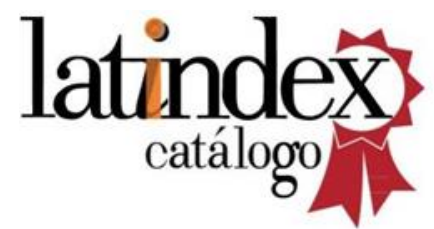

